\title{
ЗАМЕЧАНИЕ ОБ АППРОКСИМАЦИИ ИНТЕГРАЛЬНЫХ ЭКСПОНЕНТ
}

A. MARSAK. MARKUS INTEGRAALEKSPONENDI APROKSIMATSIOONI KOHTA

A. MARSHAK. A REMARK ON THE APPROXIMATION OF THE INTEGRAL EXPONENTS

\section{(Представил Г. Кузмин)}

В данной заметке результаты $\left[{ }^{1}\right]$ применяются для аппроксимации интегральных экспонент

$$
E_{p}(\tau)=\int_{0}^{1} \exp (-\tau / \mu) \mu^{p-2} d \mu ; \quad \tau \geqslant 0, \quad p=2,3, \ldots
$$

при помощи квадратурных формул (КФ)

$$
E_{p}^{n}(\tau)=\sum_{j=1}^{n} \alpha_{j} \exp \left(-\tau / \mu_{j}\right) \mu_{j}^{p-2}, \quad \tau \geqslant 0, \quad p=2,3, \ldots .
$$

Через $c$ мы будем обозначать положительные постоянные, принимающие различные значения для различных целей.

\section{1. Аппроксимация интегральных экспонент}

Обозначим через $\psi_{\tau}^{p}$ подынтегральную функцию в интеграле (1). Известно, что ее производные по $\mu$ имеют вид $\left[{ }^{2}\right]$ :

$$
\begin{gathered}
{\left[\psi_{\tau}^{p}(\mu)\right]^{(k)}=(-1)^{k+p-1}(k-p+1) ! \mu^{-k-1} \tau^{p-1} \exp (-\tau / \mu) L_{k-p+1}^{(p-1)}(\tau / \mu),} \\
k \geqslant p-1,
\end{gathered}
$$

где $L_{k}^{(\alpha)}(x)$ - обобщенный многочлен Лагерра. Функция $\psi_{\tau}{ }^{p} \in C^{\infty}$, а из (3) следует, что $\left.\left[\psi_{\tau}{ }^{p}(\mu)\right]^{(k)}\right|_{\mu=0}=0, k=p-1, p, \ldots$, однако, начиная с $k=p-1$, производные порядка $k$ не ограничены в совокупности, причем

$$
\begin{aligned}
& \left|\left[\psi_{\tau}^{p}(\mu)\right]^{(k)}\right| \leqslant c_{k, p} \mu^{p-k-2}, \quad 0<\mu \leqslant 1, \quad \tau \geqslant 0, \quad k \geqslant p-2, \\
& \left|\left[\psi_{\tau}^{p}(\mu)\right]^{(k)}\right| \leqslant c_{k, p} \tau^{p-k-2} ; \quad 0 \leqslant \mu \leqslant 1, \quad \tau>0, \quad k \geqslant p-2 .
\end{aligned}
$$

Используя неравенство «элементарной интерполяции»

$$
\min \{r, s\} \leqslant r^{\sigma} s^{1-\sigma}, \quad \sigma \in[0,1],
$$

которое верно при всех $r$ и $s \geqslant 0$, получим

$$
\left|\left[\psi_{\tau}^{p}(\mu)\right]^{(k)}\right| \leqslant c_{k, p} \mu^{-(k+2-p) \sigma} \tau^{-(k+2-p)(1-\sigma)}, \quad 0<\mu \leqslant 1, \quad \tau>0, \quad k \geqslant p-2 .
$$

Имеет место

Л е м м а 1. При всех $\tau \geqslant 0$ функция $\mu^{k+p}\left|\left[\psi_{\tau}^{p+1}(\mu)\right]^{(k)}\right|$ суммируема от 0 до 1 . 
Доказательство следует из непосредственного интегрирования с учетом асимптотических неравенств для обобщенных многочленов Лаreppa $\left[{ }^{3}\right]$.

Предположим теперь, что КФ

$$
\int_{0}^{1} f(\mu) d \mu \approx \sum_{j=1}^{n} \alpha_{j} f\left(\mu_{j}\right)
$$

$\alpha_{j} \geqslant 0, \quad j=1,2, \ldots, n, \quad 0<\mu_{1}<\mu_{2}<\ldots<\mu_{n} \leqslant 1, \quad n=1,2, \ldots, \mu_{n+1}$

A) точна для многочленов степени, непревосходящей $N$,

Б) при некотором $s \leqslant N+1$ удовлетворяет условию

$$
\sum_{j=1}^{l} \alpha_{j} \mu_{j}^{i-1}=\left(\mu_{l+1}^{i}+\mu_{l}^{i}\right) / 2 i+O\left(\mu_{1}\right) \mu_{l}^{i-1} ; \quad l=1,2, \ldots, n, \quad i=1,2, \ldots, s,
$$

В) степень сгущения узлов в направлении к левому концу отрезка интегрирования $\varrho \in[0,1)$ такова, что

$$
\mu_{j+1}-\mu_{j} \leqslant c \mu_{i 1}^{1-\rho} \mu_{j}^{\rho}, \quad j=1,2, \ldots, n .
$$

Для таких КФ, используя результаты $\left[{ }^{1}\right]$, неравенство (4) и лемму 1 , можно показать, что справедлива

Т е о р е м а 1. Пусть КФ (6) удовлетворяет условиям А) $и$ Б). Тогда для определенных в (1) и (2) функций имеет место оценка

$$
\begin{aligned}
& \max \left|E_{p}(\tau)-E_{p}^{n}(\tau)\right| \leqslant c \mu_{1}^{p-1} \times \\
& \times \begin{cases}1+x_{N}(s, t), & \left\{\begin{array}{l}
2 p-3=t \leqslant N-s+1, s_{1} / 2<s<N, \\
2 p-3<t \leqslant N-s+1,0<s<N,
\end{array}\right. \\
1+x_{N}(s, t)\left|\ln \mu_{1}\right|, & \left\{\begin{array}{l}
2 p-4=t \leqslant N-s+1 ; s_{1} \leqslant s<N, \\
2 p-3=t \leqslant N-s+1 ; s_{1} / 2=s<N,
\end{array}\right.\end{cases}
\end{aligned}
$$

где $s_{1}=1 /(1-0)$, параметр @ определен в $\left.\mathrm{B}\right)$, а функция

$$
\varkappa_{N}(s, t)=\mu_{1}^{-t / 2} /(N-s)^{t} .
$$

\section{2. Поточечная и интегральная оценки}

Т е о р ем а 2. Пусть выполнены условия теоремы 1. Тогда для любого $\sigma \in(1 / 2,1], \sigma \neq \mathrm{Q}$ справедливы оценки

$$
\begin{aligned}
& \left|E_{2}(\tau)-E_{2}^{n}(\tau)\right| \leqslant c \mu_{1}\left(\frac{\mu_{1}}{\tau}\right)^{s(1-\sigma)}+c_{1} \mu_{1} \varkappa_{N}(s, t) \cdot \times
\end{aligned}
$$

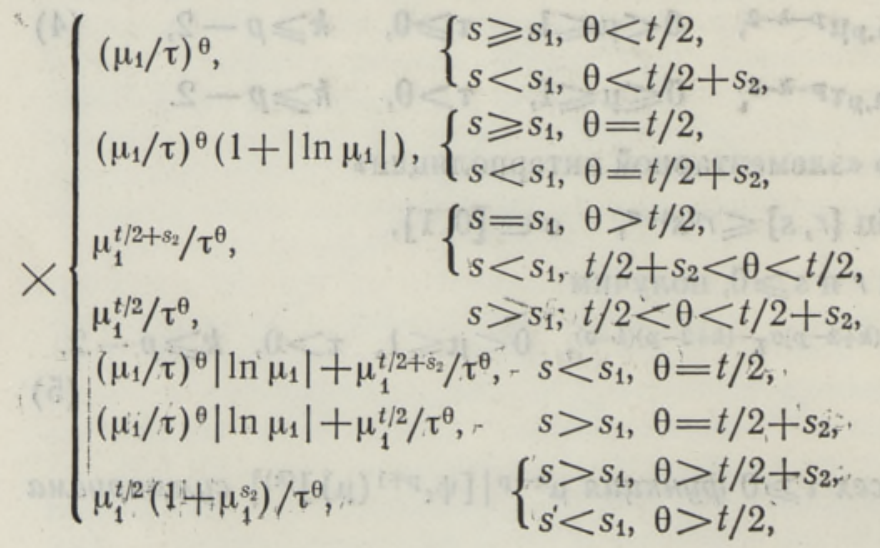


где $s_{2}=s(1-\varrho)-1, \theta=(s+t)(1-\sigma)$, а функция $x_{N}$ определена в $(8)$. Здесь $s$ и $t$ целье, причем $0<s<N, 0 \leqslant t \leqslant N-s+1$.

Доказательство этой теоремы аналогично доказательству теоремы 1 , но для оценки производных от функции $\psi_{\tau}^{2}$ вместо неравенства (4) применяется неравенство (5).

Следующая теорема дает нам интегральную оценку.

Т е о рем а 3. Пусть выполнены условия теоремы 1, где в условии Б) $s=s_{1}$. Тогда, если $\left|\ln \mu_{1}\right| /(N-s) \rightarrow 0, n \rightarrow \infty$, то справедливо равенство

$$
\int_{0}^{b}\left|E_{2}(\tau)-E_{2}^{n}(\tau)\right| d \tau=o\left(\mu_{1}\right), \quad b=\text { const }<\infty .
$$

Д ок а за те льс т в о. Разбивая отрезок интегрирования на две части - от 0 до $\mu_{1}$ и от $\mu_{1}$ до $b$, и применяя к первому интегралу оценку (7), а ко второму оценку (9) при $s=s_{1}$, придем к неравенству (10).

3 а м е ч а н и е. При $0 \leqslant \varrho<1$ и $1 / 2<\sigma<1, \sigma \neq \varrho$ введем целочисленные множества

$$
L_{1}(\varrho, \sigma)=\left\{(s, t): 1<\theta<t / 2, s_{1}<s<N, 0 \leqslant t \leqslant N-s+1\right\},
$$

$L_{2}(\varrho, \sigma)=\left\{(s, t): 1<\theta<t / 2+s_{2} ; 0<s<\min \left\{s_{1}, N\right\}, 0 \leqslant t \leqslant N-s+1\right\}$.

Тогда в условиях теоремы 1 , если $(s, t) \in L_{1}(\varrho, \sigma)$ или $(s, t) \in$ $\in L_{2}(\varrho, \sigma), 0 \leqslant \varrho<1,1 / 2<\sigma<1, \sigma \neq \varrho$, справедлива более точная, чем (10), оценка

$$
\int_{0}^{b}\left|E_{2}(\tau)-E_{2}^{n}(\tau)\right| d \tau \leqslant c \mu_{1}^{2}\left(1+\chi_{N}(s, t)\right),
$$

где $\varkappa_{N}$ определена в (8).

\section{Л ИТЕР А Т У Р А}

1. Мариак А. Изв. АН ЭССР. Физ. Матем., 35, № 4, 390-399 (1986).

2. Марцак А. Л. О скорости сходимости метода дискретных ординат решения уравнения переноса. Автореф. дис. канд. фнз.-матем. наук. Новосибирск, 1983.

3. Суэтин П. К. Классические ортогональные многочлены. М., «Наука», 1979.

Институт астрофизики и физики атмосферы Академии наук Эстонской ССР
Поступила в редакцию 11/V 1986 